\title{
Analysis and Design of an Effective Light Interference Methane Sensor Based on Three-Dimensional Optical Path Model
}

\author{
Teng Long, ${ }^{1,2}$ En Li $\mathbb{D}^{1,2}$ Lei Yang, ${ }^{1,2}$ Junfeng Fan, ${ }^{1,2}$ and Zize Liang ${ }^{1,2}$ \\ ${ }^{1}$ The State Key Laboratory of Management and Control for Complex Systems, Institute of Automation, Chinese Academy of Sciences, \\ 95 Zhongguancun East Road, Beijing 100190, China \\ ${ }^{2}$ University of Chinese Academy of Sciences, No. 19(A) Yuquan Road, Beijing 100049, China
}

Correspondence should be addressed to En Li; en.li@ia.ac.cn

Received 12 December 2017; Revised 24 May 2018; Accepted 4 June 2018; Published 10 July 2018

Academic Editor: María del Carmen Horrillo

Copyright (c) 2018 Teng Long et al. This is an open access article distributed under the Creative Commons Attribution License, which permits unrestricted use, distribution, and reproduction in any medium, provided the original work is properly cited.

As an important environmental monitoring equipment, the existing methane sensors or the traditional interferometer-based methane detectors have some drawbacks, such as low accuracy, large size, and complex calibration operations. Moreover, the optical path model and analysis method for the light interference methane sensor are not practical. In this paper, an effective light interference methane sensor is proposed based on a three-dimensional optical path model with point light source. Based on this model, the interference optical system is studied to illustrate the cause of the interference fringes. Furthermore, the influencing factors of the light intensity distribution are analyzed and an adjustment method for the interference fringes is proposed, which helps to simplify the assembling and calibrating operations. In order to improve the measurement accuracy, a temperature drift compensation method which includes a mapping table, a steady-state compensator, and a dynamic compensator is proposed. The mapping table is established between the output voltages of photoelectric detector, and the methane concentration, the steady-state compensator, and the dynamic compensator are proposed to eliminate the temperature drift. Finally, an experimental device for the light interference methane sensor is constructed to validate the interference fringe adjustment method and the temperature drift compensation method.

\section{Introduction}

Methane is widely distributed in nature; it is the main component of natural gas, oil gas, and coal mine gas. Methane can be made artificially, and it is an important clean energy. But the methane concentration in the air needs to be strictly controlled. If the methane concentration in the air exceeds $5 \% \sim 15 \%$, it is very flammable and may cause violent explosions under suitable conditions. If the methane concentration in the air reaches $25 \% \sim 30 \%$, it can cause some uncomfortable symptoms, such as headache, dizziness, fatigue, heartbeat acceleration, ataxia, and even death of asphyxia. Therefore, it is necessary to monitor methane concentration in real time in special environment, such as gas fueling station, coal mine, gas treatment plant, and various kinds of pipelines.
According to the detection principle for methane, the methane sensors are divided into various categories such as catalytic combustion methane sensor, thermal conductivity methane sensor, gas sensitive semiconductor methane sensor, infrared methane sensor, and light interference methane sensor [1]. Catalytic combustion methane sensor uses the resistance of the detection element to reflect the heat that is produced by the oxidation-reduction reaction $[2,3]$. The thermal conductivity methane sensor makes use of the difference in thermal conductivity between methane and air to obtain the electrical signals $[4,5]$. The detection principle of gas sensitive methane sensor is that the resistivity of metal oxide changes significantly when the metal oxide is adsorbed on different gases [6-8]. The principle of infrared methane sensor is that different gases have different absorption spectra of infrared radiation, and the intensity of absorption is 
related to the methane concentration [9-11]. However, these methane sensors do not measure methane concentration in large scale and require frequent calibration.

The light interference methane sensor uses the principle of light interference to measure the methane concentration [12]. The refractive index of coherent light is different in different media because of which the optical path difference varies, leading to the movement of interference fringes [13, 14]. Light interference methane sensor has many advantages such as high sensitivity, wide measurement range, and good stability. It is gaining popularity and is being increasingly used in mining. However, some problems have been found during the process of using the methane sensor. For example, assembly difficulties, interference fringes spacing cannot be adjusted, measurement accuracy is susceptible to temperature. It is necessary to establish an accurate optical path model to analyze the influencing factors of the measurement accuracy.

In 2014, Lin improved the traditional optical path structure of the light interference methane sensor by reducing the size of the sensor $[15,16]$. The planar optical path model is used to analyze the principle of light interference methane sensor. However, there are two hypotheses for the planar optical path model. First, it is assumed that the incident light of the interference methane sensor is parallel light, and the angle between incident light and refracting prism in the vertical direction is 90 degrees. This hypothesis can be used to explain the measurement principle of light interference methane sensor, but it cannot explain the cause of interference fringes. Second, it is assumed that the optical path on the horizontal plane is exactly the same, and the light path is only analyzed on a horizontal plane. However, in the actual optical interference methane sensor system, the angle of each incident light is different. Only by analyzing the difference of the optical path of different planes, the regulation of the interference fringes can be obtained. On the other hand, the optical measurement part requires very high stability, and the temperature has a great influence on the optical devices and mechanical parts. So, the light interference methane sensor is particularly sensitive to the temperature, and it is easy to produce temperature drift. Therefore, the design of a general temperature compensation method is the premise for accurate measurement of the light interference methane sensors.

Based on the light interference methane sensor proposed by Lin [15], we have accomplished the following. Firstly, we proposed a three-dimensional optical path model with point source. This model could explain the causes of interference fringes and analyze the influencing factors of the fringe spacing. Secondly, we proposed a method for adjusting the interference fringes and a temperature drift compensation method. Finally, we have designed an experimental setup and verified the validity of the methods.

The rest of the paper is organized as follows: In Section 2, the three-dimensional optical path model is proposed. In Section 3, the propagation of the optical path and the adjustment of interference fringes based on the point source light interference methane sensor are described in detail. In Section 4, the factors that affect the fringe spacing of interference fringes are analyzed, and the temperature drift compensation method is proposed to reduce the measurement error. In Section 5 and Section 6, the experimental setup and experimental results are discussed to verify the effectiveness of the proposed three-dimensional optical path model and the temperature drift compensation method. Finally, the main contents of this paper are summarized.

\section{Three-Dimensional Optical Path Model}

In our previous work, the traditional light interference methane sensor was improved to reduce the size of the optical system and it was suitable for various measuring environments [15]. The original three chambers were changed to two chambers. The planar optical model is shown in Figure 1. However, in the planar optical path model that we used before, parallel light was incident on the refracting prism and the optical paths of each parallel light were consistent because of which the interference fringes could not be explained.

In the actual optical structure, the light source cannot emit a parallel light, and some of the light must form a certain angle with the reflecting surface of the refracting prism.

Assuming that the length of the chamber is $L$, the refractive index of air in the reference chamber is $n_{r}$, and the refractive index of gas to be measured in the sample chamber is $n_{s}$. If the interference fringes are generated, the intensity distribution of the interference fringes could be described as follows [17]:

$$
I_{r}=2 I_{0}\left(1+\cos \left(\frac{2 \pi \cdot \Delta}{\lambda}\right)\right),
$$

where $\lambda$ is the wavelength of the incident light, $I_{r}$ is the intensity of the interference fringes, and $I_{0}$ is the intensity of the incident light. $\Delta=\left(n_{s}-n_{r}\right) L$ is optical path difference.

We assume that the light source is a point light source, and a radial light is formed after passing through a narrow gap. In the three-dimensional optical path model, the influence of the chamber is consistent with that in the planar optical path model. The three-dimensional optical path model is shown in Figure 2.

There is a difference in the light propagation along different planes, because the beam emitted by a point source is not parallel. As shown in Figure 2, the light $L_{1}$ is obliquely injected into the refracting prism, and the angle between the incident light plane and the reflecting plane of the refracting prism is $45^{\circ}$. The refracting prism that uses the detachment amplitude method to obtain the light $L_{2}$ and $L_{3}$ is shown in Figure 2. $L_{1}, L_{2}$, and $L_{3}$ are in a plane, and $L_{2}$ and $L_{3}$ are parallel to each other and spreading obliquely. $L_{2}$ and $L_{3}$ pass through the triangular reflecting prism and form $L_{4}$ and $L_{5} . L_{4}$ and $L_{5}$ form new planes, after which they are superimposed by the refracting prism to produce the interference fringes. 


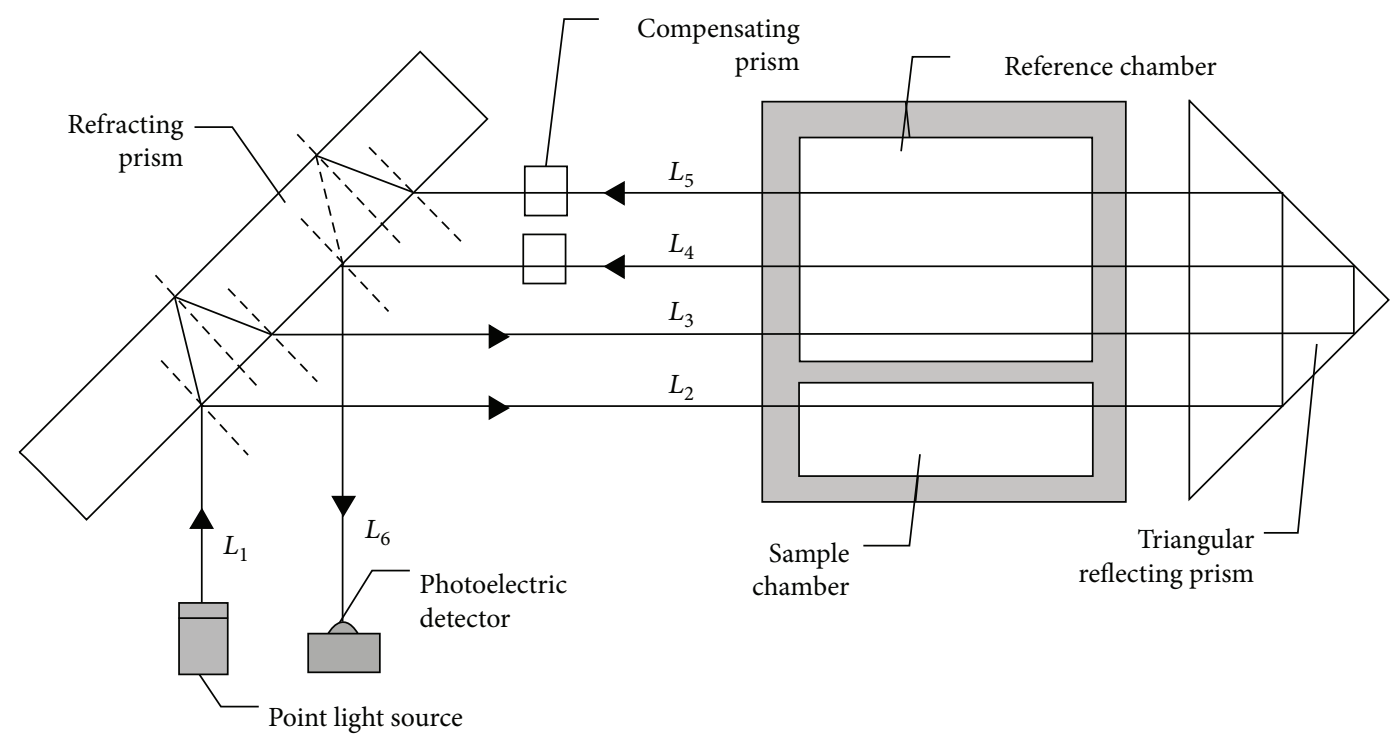

FIgURe 1: Planar optical model.

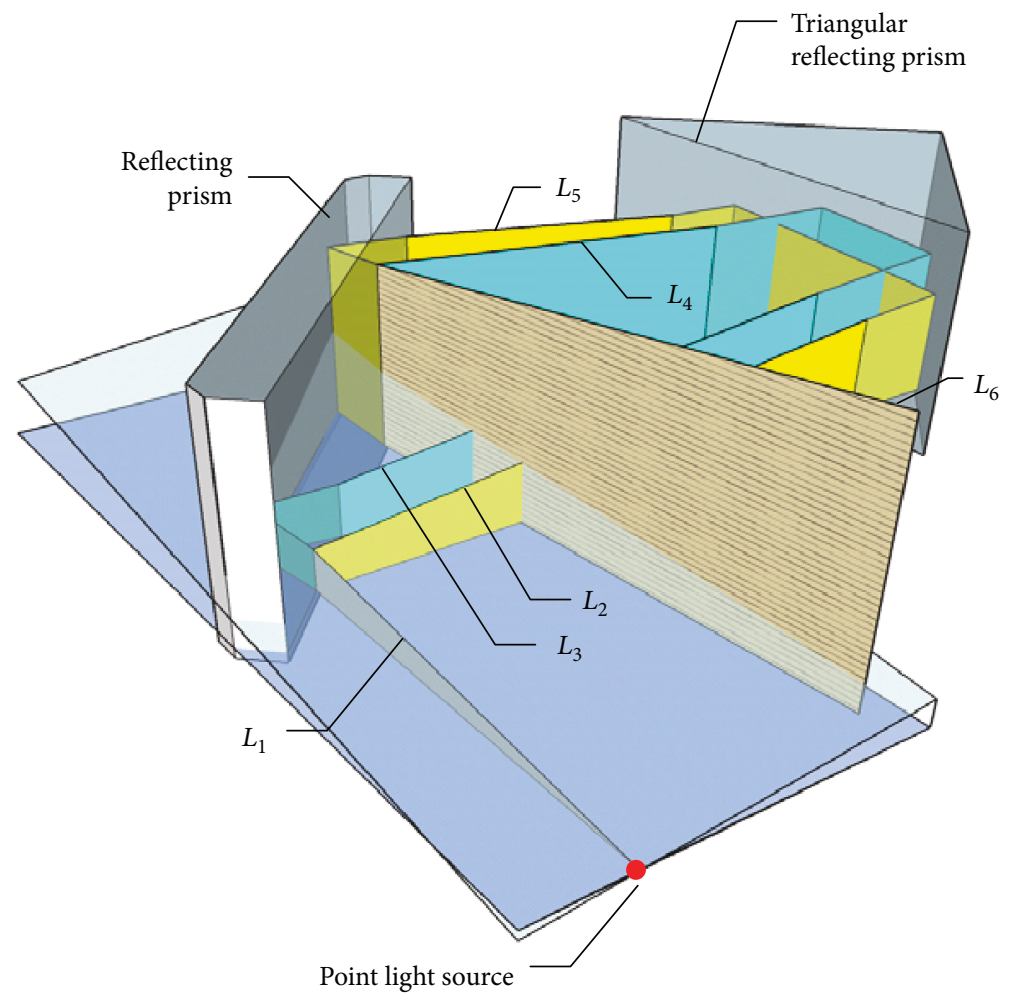

FIgURE 2: Three-dimensional optical path model.

\section{Light Propagation and Adjustment of Interference Fringes}

The light from the point light source passes through the refracting prism at different angles, and $L_{2}$ and $L_{3}$ will also pass through the triangular reflecting prism at different angles. Therefore, different incident angles cause different propagation distances of light, which eventually results in an interference fringe in the vertical direction of the photoelectric detector. We analyzed the incident light at two different angles in the same vertical plane.

The propagation of light on the top view and profile view is discussed through orthogonal decomposition. If the position of the refracting prism and the triangular reflecting prism is fixed, the light remains unchanged in the top view regardless of the different incident angles. As shown in Figures 3(a) and 3(b), the optical path in the top view of the three-dimensional optical model is same as the optical 


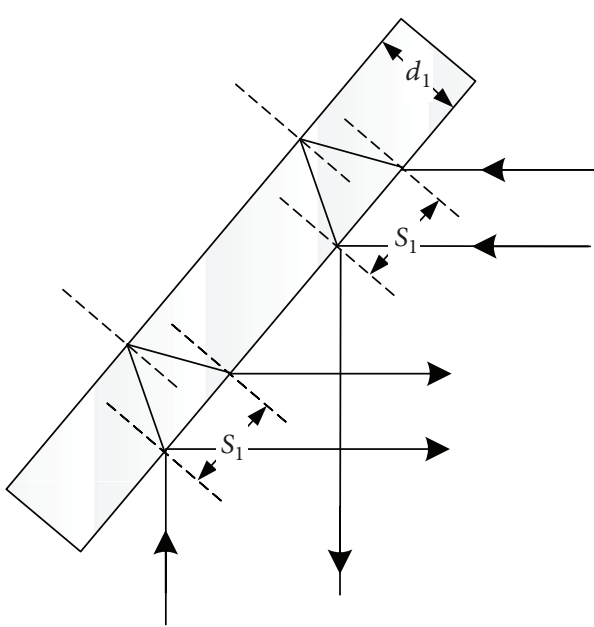

(a)

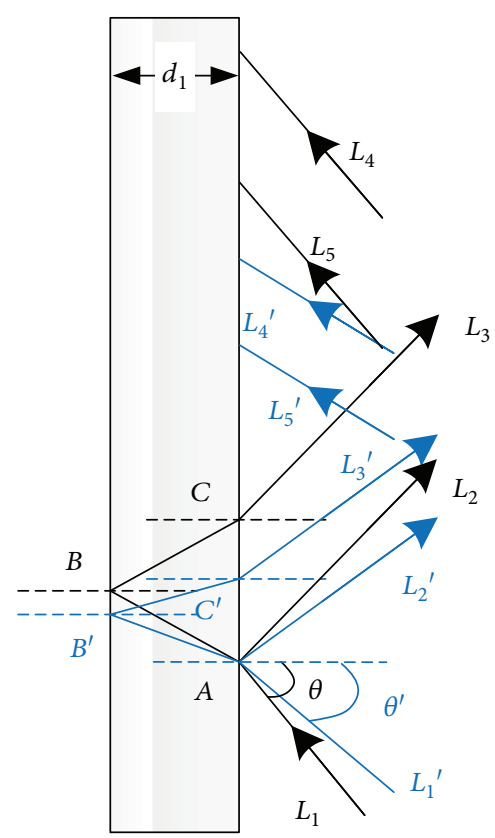

(c)

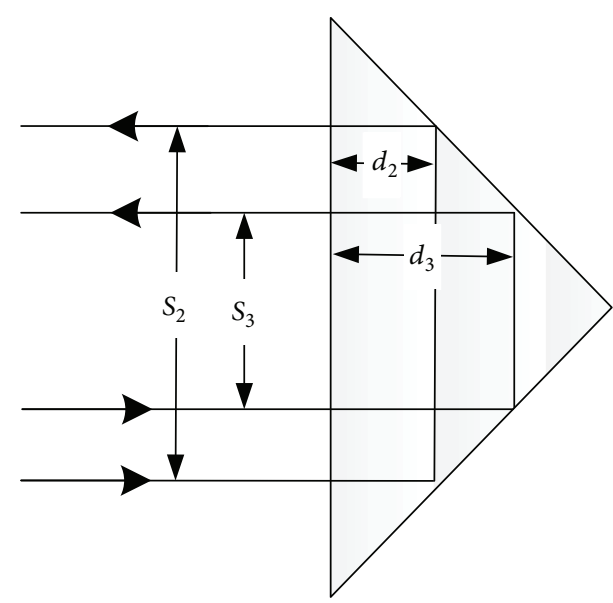

(b)

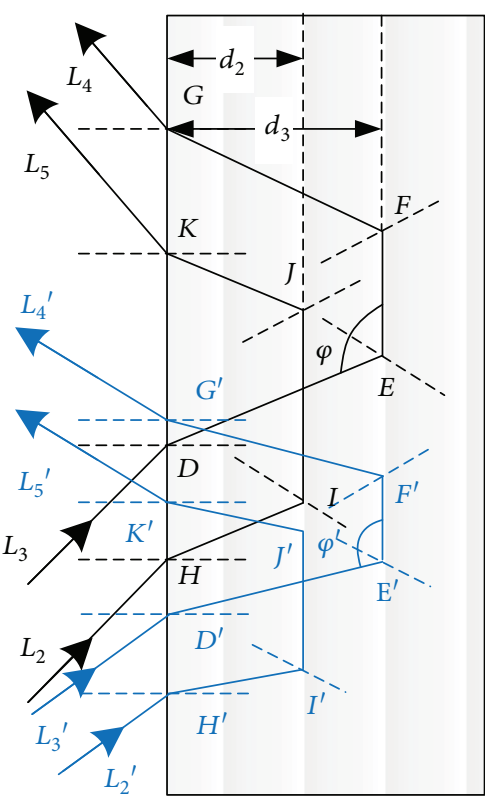

(d)

FIGURE 3: (a) Optical path of the refracting prism in top view, (b) optical path of the triangular reflecting prism in top view, (c) optical path of the refracting prism in profile view, and (d) optical path of the triangular reflecting prism in profile view.

path in the planar optical path model. $S_{1}, S_{2}$, and $S_{3}$ represent the distances between the optical paths. $d_{1}$ represents the thickness of refracting prism; $d_{2}$ and $d_{3}$ represent the distance between the reflection point and the incident plane in the triangular reflecting prism.

The incident light in the profile view at two different incidence angles is shown in Figures 3(c) and 3(d). $\theta$ and $\theta^{\prime}$ represent the incident angle of incident light $L$ and $L^{\prime}$ in a vertical plane. $\varphi$ and $\varphi^{\prime}$ represent the angle between incident light and reflected light on the vertical plane when light $L_{2}$ reflects for the first time in the triangular reflector prism. Only the optical path of the incident light from the light source is fully drawn in Figure 3(c). The light $L_{4}$ and $L_{5}$ is similar to the optical path of $L_{1}$. The optical path of the profile view in the triangular reflecting prism is shown in Figure 3(d).
3.1. Single Light Propagation. According to the geometric relationship between Figures 3(a) and 3(c), when $L_{1}$ passes through the refracting prism, the optical path difference can be described as follows:

$$
\begin{aligned}
\Delta_{1} & =n_{p}\left(\sqrt{l_{A B}^{2}+\frac{S_{1}^{2}}{4}+d_{1}^{2}}+\sqrt{\frac{S_{1}^{2}}{4}+l_{B C}^{2}+d_{1}^{2}}\right) \\
& =n_{p} \sqrt{4 l_{A B}^{2}+S_{1}^{2}+4 d_{1}^{2}},
\end{aligned}
$$

where $n_{p}$ represents the refractive index of light in the prisms, $l_{A B}^{2}$ represents the distance between $A$ and $B$ points, and $l_{B C}^{2}$ represents the distance between $B$ and $C$ points.

When $L_{4}$ and $L_{5}$ pass through the reflecting prism, the optical path difference can be described as follows: 


$$
\Delta_{2}=-n_{p} \sqrt{4 l_{A B}^{2}+S_{1}^{2}+4 d_{1}^{2}} .
$$

Therefore, coherent light propagating in the refracting prism does not produce an optical path difference.

When $L_{2}$ and $L_{3}$ pass through the triangular reflecting prism, two isosceles trapezoids $H I J K$ and DEFG are generated in the profile view. Because $L_{2}$ and $L_{3}$ are injected in parallel into the triangular reflecting prism and ejected in parallel, the geometric relationship can be described as follows:

$$
l_{H K}=l_{D G}, H I\|D E, K J\| G F .
$$

If the baselines of the two isosceles trapezoids coincided with each other, we could obtain the geometric relation shown in Figure 4.

According to the geometric relation shown in Figure 4, if $l_{F J}=l_{J O}$, then $\varphi=\pi$ and $\theta=0$. When the light incident angle $\theta$ is 0 in the profile view, the optical path difference of the coherent light in the triangular reflecting prism is 0 . When $\theta \neq 0$, the optical path difference in the profile view of the triangular reflecting prism can be described as follows:

$$
\begin{aligned}
\Delta_{3}^{\prime} & =2 n_{p}\left(l_{F J}-l_{J O}\right)=2 n_{p}\left(d_{3}-d_{2}\right) \frac{1+\cos \varphi}{\sin \varphi} \\
& =2 n_{p}\left(d_{3}-d_{2}\right) \cot \frac{\varphi}{2} .
\end{aligned}
$$

According to the geometric relation of space and (5), the optical path difference in the triangular reflecting prism can be described as follows:

$$
\Delta_{3}=\sqrt{4\left(n_{p}\left(d_{3}-d_{2}\right) \cot \frac{\varphi}{2}\right)^{2}+\left(S_{2}-S_{3}\right)^{2}} .
$$

3.2. Multiple Light Propagation. An interference fringe is formed at the photoelectric detector, and it must be composed of several lights that have different light intensities. According to (1), the different light intensities could be obtained by changing the incident light intensity, the incident wavelength, and the optical path difference. The light interference methane sensor in this paper is a fixed light source, and it cannot change the intensity and wavelength of each incident light. Therefore, the interference fringes are formed by changing the optical path difference of coherent light.

As shown in Figure 3(c), $L_{1}$ and $L_{1}^{\prime}$ are the two incident lights, which have different directions. The incident angle of $L_{1}$ is greater than that of $L_{1}^{\prime}$ in the profile view. According to (2) and (3), the optical path difference in the refracting prism is compensated when the incident light traverses back. Therefore, the optical path difference in the refracting prism is 0 regardless of the incident angle. Meanwhile, the incident angles of $L_{3}^{\prime}$ and $L_{4}^{\prime}$ are less than $L_{3}$ and $L_{4}$ due to the difference in the incident angle $\theta$ and $\theta^{\prime}$. Therefore, $\varphi^{\prime}$ is less than $\varphi$, as shown in Figure 3(d). According to Figure 4 and (6), when $\varphi$ reduces to $\varphi^{\prime}$, the value of $\cos \varphi$ increases, which results in the increase in optical path difference in the

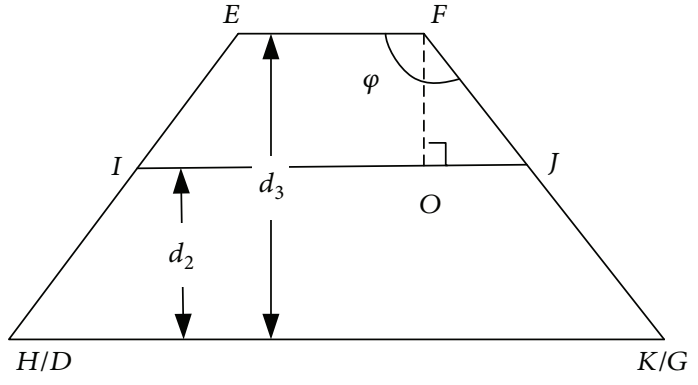

FIGURE 4: Geometrical relation of the optical path in the triangular reflecting prism.

triangular reflecting prism. According to the optical principle, the value of $\cos \varphi=-\sin \theta / n_{p}$.

As mentioned above, the light intensity distribution detected by the photoelectric detector is related to $\theta, \varphi, d_{2}$, $d_{3}, S_{2}$, and $S_{3}$. If the point light source and the position of the prisms are fixed, the optical path difference of each light is determined by the incident angle $\theta$ of the profile view. Each incident light emitted from the point light source has different angles and remains constant. Therefore, a stable interference fringe is formed in the photoelectric detector.

3.3. Adjustment of Interference Fringe Spacing. The methane sensor measures the methane concentration in the sample chamber by detecting the movement of the interference fringes; therefore, it is necessary to ensure that the interference fringe spacing is equal to the height of the photoelectric detector in the vertical direction. The position relation between the interference fringes and the photoelectric detector is shown in Figure 5.

In the optical path system, the light intensity distribution on the photoelectric detector is directly related to the optical path difference. In order to keep the optical path system stable, the positions of the light source, the refracting prism, and the triangular reflecting prism are kept fixed. Therefore, a compensating prism is added to the optical path system to adjust the interference fringe spacing. As shown in Figure 6, the compensating prism consists of two cuboid prisms, $p_{1}$ and $p_{2}$, which form an included angle $\gamma . L_{4}$ and $L_{5}$ pass through prisms $p_{1}$ and $p_{2}$, respectively, and the optical path difference of $L_{4}$ and $L_{5}$ is changed. The same phenomenon occurs with $L_{4}^{\prime}$ and $L_{5}^{\prime}$. According to the geometric relation, the optical path difference between $L_{4}$ and $L_{5}$ in the compensating prism is described as follows:

$\Delta_{p}=M Q-N P=x n_{p}\left(\frac{1}{\sqrt{n_{p}^{2}-\sin ^{2} \beta}}-\frac{1}{\sqrt{n_{p}^{2}-\sin ^{2}(\beta-\gamma)}}\right)$,

where $\beta$ is the incidence angle of $L_{4}$, and $x$ is the width of the cuboid prism.

Because the light from the point source is radial, there must exist an incident light with incident angle 0 in 


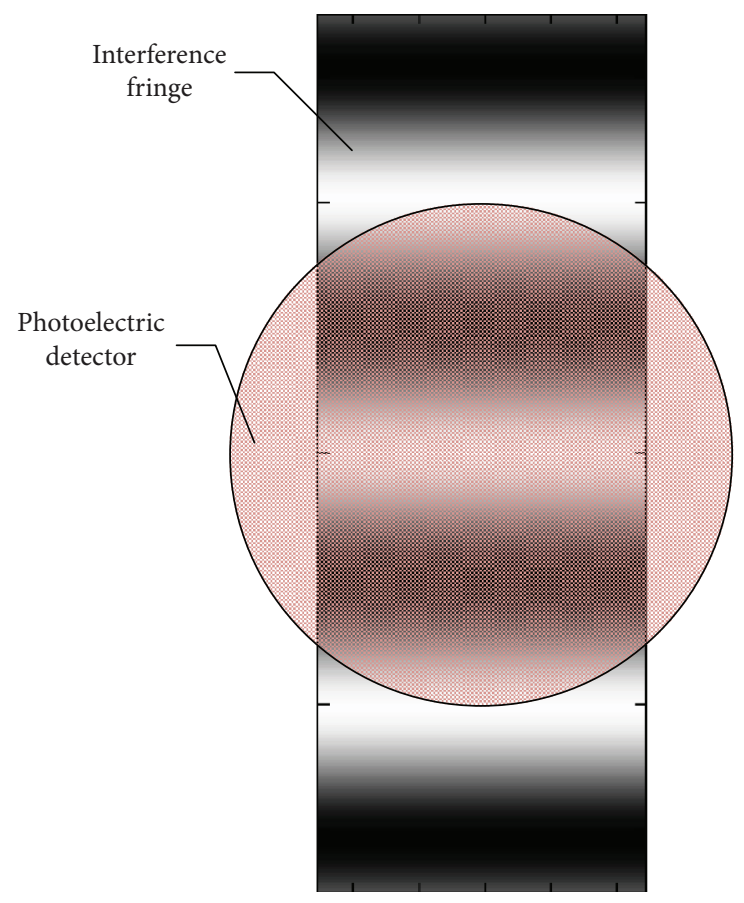

FIgURE 5: Relative position relation between interference fringes and photoelectric detector.

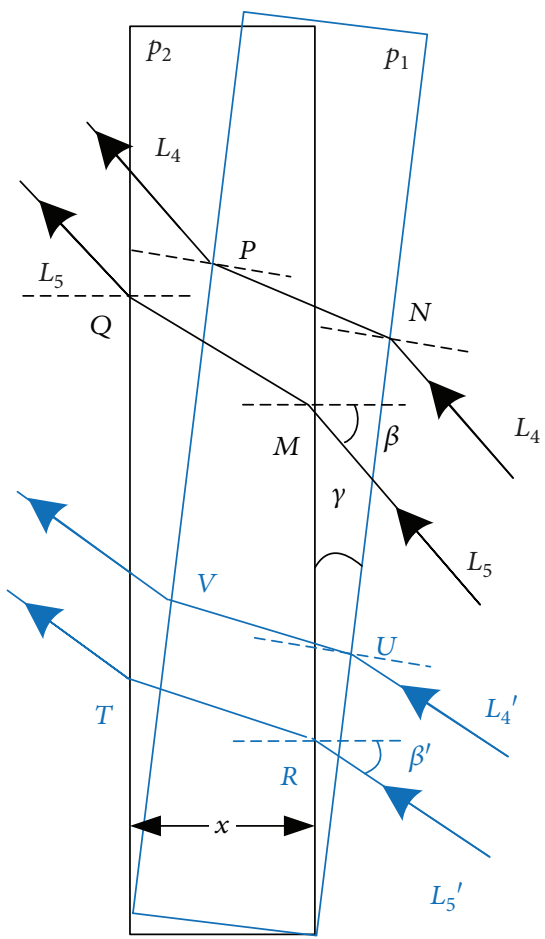

FIgURE 6: Optical path of the compensating prism.

Figure 3(c), and $\beta$ in Figure 6 is 0 . When $\beta=0$, the optical path difference between $L_{4}$ and $L_{5}$ is described as follows:

$$
\Delta_{p 0}=x\left(1-\frac{n_{p}}{\sqrt{n_{p}^{2}-\sin ^{2}(-\gamma)}}\right) .
$$

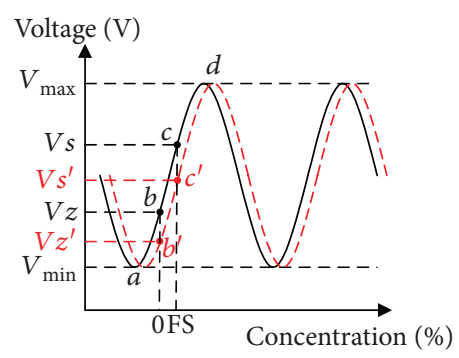

FIGURE 7: Relationship between methane concentration and output voltage of the photoelectric detector.

Due to $\Delta_{p} \neq \Delta_{p 0}$, the compensating prism has different effects on the light at different incident angles given by $\theta$. According to the actual optical path, $\gamma \cong 0$ and $\Delta_{p 0} \cong 0$. Therefore, the increment in the optical path difference is monotone similar to $\theta$, leading to a change in the interference fringe spacing.

\section{Temperature Drift Compensation Method}

According to the optical path described in Section 3, many factors will affect the measurement accuracy. In addition to the methane concentration in the sample chamber, the pitching angle of the light source, the pitching angle of the refracting prism, and the included angle of the compensating prism also affect the spacing or light intensity distribution of the interference fringes. The point light source and all the optical devices are fixed on the shell by screws to maintain the stability of the optical path system. Nevertheless, the shell will cause a small deformation due to temperature changes, and the refractive index of the prism will vary with temperature. In addition, the wavelength of the light source, the characteristics of the photoelectric detector, and the amplification processing circuit will be affected by the temperature. All these factors will lead to the change in the interference fringe shifting or the interference fringe spacing. Therefore, temperature is a crucial factor affecting the accuracy of the sensor, and the sensor temperature compensation is mainly considered in this paper.

4.1. Steady-State Compensator. The relationship between measurement errors and temperature variation is complex, and there are differences between each sensor. Therefore, it is necessary to design a general method to compensate the temperature drift of methane sensors. The output voltage curve of photoelectric detector with the methane concentration is shown in Figure 7. There is a sinusoidal relationship between the output voltage and methane concentration because of which the maximum measurement range is the difference between the maximum and the minimum of the sinusoidal curve. In order to distinguish the repeated voltage in the sinusoidal curve, the light interference methane sensor needs to be calibrated before measurement, and the highest concentration in the environment should be limited. In order to obtain an approximate linear measurement curve, we reduce the measurement range by selecting one-third segment of the half period of the sinusoid for measurement. 
As shown in Figure 7, the $b c$ segment is taken as the measuring curve and calibrated, point $b$ is the zero point of the measurement range, and point $c$ is the full range point of the measurement range. Therefore, the measurement curve is approximately a straight line. When the temperature is changed, the sinusoidal curve will move and the variation is shown as the red dotted line in Figure 7, where $b$ and $c$ move down to $b^{\prime}$ and $c^{\prime}$. In this paper, an effective steady-state temperature drift compensation method is proposed to reduce error caused by temperature drift and improve the accuracy of the steady-state measurement.

To realize the steady-state temperature compensation, the sensor is placed in the temperature control box, and the temperature is varied between $-10^{\circ} \mathrm{C}$ and $40^{\circ} \mathrm{C}$. The zero point drift value $C 0_{i}$ and full range point drift value $C f_{i}$ under temperature $T_{i}$ are recorded. According to our analysis and verification, $T_{i}$ is $-10^{\circ} \mathrm{C}, 0^{\circ} \mathrm{C}, 10^{\circ} \mathrm{C}, 20^{\circ} \mathrm{C}, 30^{\circ} \mathrm{C}$, and $40^{\circ} \mathrm{C}$, respectively. The piecewise linear compensation for temperature drift and calibration of the measuring curve slope is realized. This compensation process is used as a steady-state compensator for methane sensors. The steadystate compensator could be described as follows:

$$
\begin{aligned}
& C_{s}=\frac{\left(C^{\prime}-C 0\right) * F S}{C f-C 0}, \\
& C 0=\frac{\left(T-T_{i}\right)\left(C 0_{i+1}-C 0_{i}\right)}{T_{i+1}-T_{i}}+C 0_{i}, \\
& C f=\frac{\left(T-T_{i}\right)\left(C f_{i+1}-C f_{i}\right)}{T_{i+1}-T_{i}}+C f_{i},
\end{aligned}
$$

where $C_{s}$ is the methane concentration after the steady-state compensation, $T$ is the current temperature, and $F S$ is the measurement range of the sensor.

4.2. Mapping Table. When the temperature drift is too large, the measured curve will be located in the nonlinear measurement range. Therefore, the assumption that the output voltage varies approximately linearly with the methane concentrations is untenable. It will seriously affect the accuracy of the measurement.

To solve the problem of the $b c$ segment in the nonlinear segment, the output voltage value of the photoelectric detector and the methane concentration value that is compensated by the steady-state compensator are recorded. The linear functions are fitted between two points of the mapping table. The concentration range of the mapping table is the concentration range corresponding to the maximum range of the output voltage at ambient temperature. The error caused by the measurement curve in the nonlinear segment can also be reduced by the mapping table. Because the methane concentration is nonnegative, the standard methane sample cannot be used to obtain a concentration below $0 \%$. However, the calculation results may be negative during data processing. Therefore, the pressure method is used to simulate the methane concentration and a complete mapping table is established. The pressure method uses the pressure difference between the reference chamber and the sample chamber to simulate the corresponding methane concentration. The relationship of methane concentration $c_{m}$ and pressure difference $p$ could be described as $c_{m}=p / 0.518$.

4.3. Dynamic Compensator. Only the influence of stable temperature is considered in the steady-state compensator, but the influence of temperature change on measurement accuracy is not considered. Because temperature is a very large lag factor, the ambient temperature, internal temperature of the sensor, and the temperature of the electronic components are not exactly the same, because of which the steady-state compensator cannot eliminate the drift in the dynamic process. In the actual experiment, it is also observed that the zero drift is very severe during the temperature change.

A dynamic compensator is designed in this paper. The internal and external temperature of the methane sensor is collected at the same time. A linear line is fitted to compensate the temperature in the dynamic process by establishing the relationship between the temperature difference and the zero point drift value. After the compensation, the methane concentration is expressed as follows:

$$
\begin{gathered}
c(t)=C_{0}(t)-C_{s}-C_{d}, \\
C_{d}=k \Delta T(t)+b,
\end{gathered}
$$

where $C_{0}(t)$ is the methane concentration in the sample chamber before the temperature compensation, $k, b$ are the dynamic compensation constants, and $\Delta T(t)$ is the temperature difference.

The measurement process of the light interference methane concentration sensor is shown in Figure 8, which summarizes the abovementioned steps.

Figure 8 depicts the measurement process of the methane sensor using a point light source. The point light source forms a diffused surface light source when the light passes through a narrow slit. After the light passes through the refracting prism, the chambers, and the triangular reflecting prism, interference fringes are formed at the photoelectric detector, which measures the movement of the interference fringe and outputs the corresponding voltage value. By establishing the mapping table between the voltage and the methane concentration, the sinusoidal curve is mapped into approximate linear curve and the measurement error caused by the nonlinear segment in the sinusoidal curve can be eliminated to the maximum extent possible. After the mapping of the voltage value, the temperature drift compensation method is used to obtain the methane concentration.

\section{Experimental Setup}

In order to verify the effectiveness of the proposed threedimensional optical path model of the light interference methane sensor based on a point light source, a practical light interference methane concentration sensor system was implemented in this study, as shown in Figure 9(a). A monochromatic LED was used as the point source, and the LED was packaged in housing with a narrow slit so that the point source was only a beam of light in the 


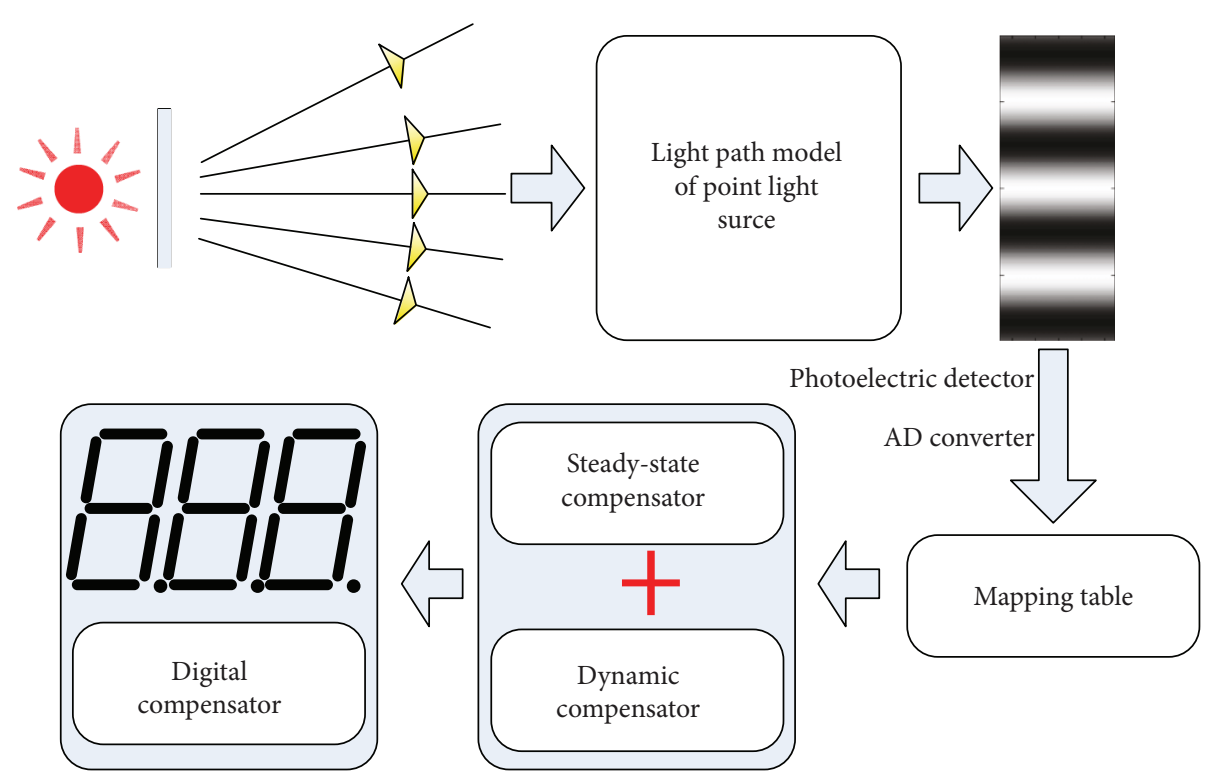

FIGURE 8: Flow chart of the light interference methane sensor with a point light source.

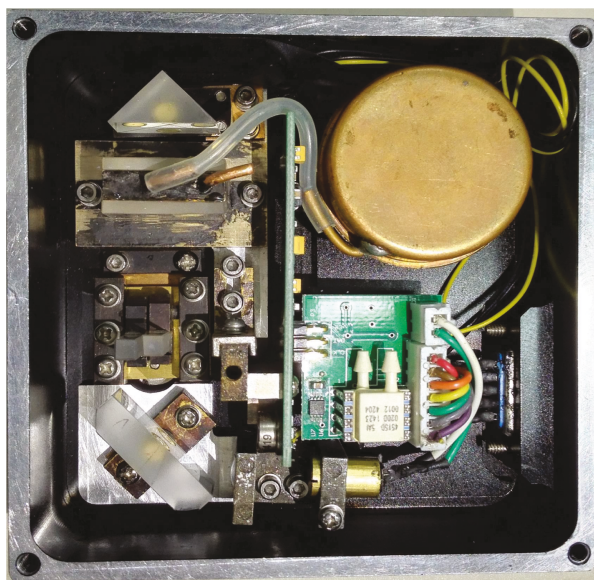

(a)

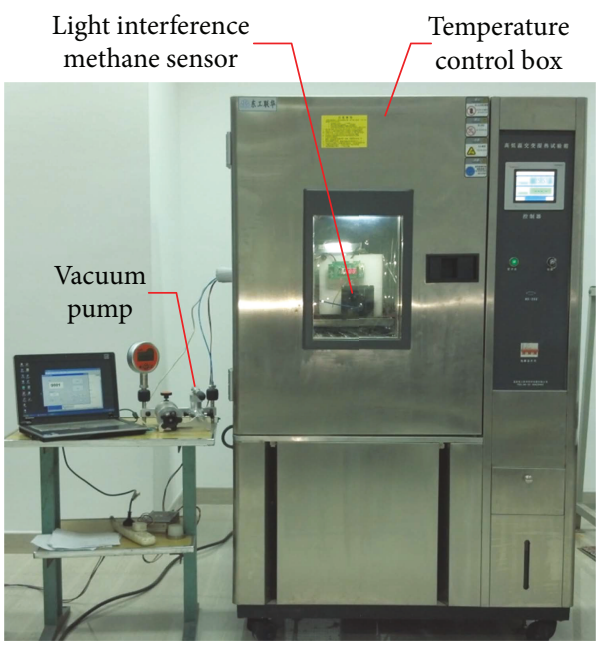

(b)

FIGURE 9: (a) Light interference methane sensor and (b) experimental system.

vertical direction. A refracting prism, a triangular reflecting prism, a compensating prism, a shell, and some supporting brackets were installed. One end of the compensating prism was connected to the shell and the other end was connected to a screw, which made it convenient to adjust the included angle $\gamma$ in the outer side of the shell. The light intensity of the interference fringes was detected by the photoelectric detector, and the output value of the voltage was used to reflect the movement of the fringe. After the analog-to-digital sampling, the microprocessor was used to process the data and send it to the upper computer to analyze the data.

The experimental device was designed based on the relation between the thickness of the chamber and the measurement range [15]. The detection limit is only related to the length of the gas chamber. The relation between the length of the gas chamber and the measuring range can be expressed as follows:

$$
L=\frac{s \lambda}{\left(c^{\prime}-c\right) \%\left(n_{\mathrm{CH}_{4}}-n_{\mathrm{AIR}}\right)},
$$

where $\lambda$ is the wavelength of the incident light, $L$ is length of the gas chamber, and $s$ is maximum detectable displacement of interference fringe. $c^{\prime}-c$ is maximum concentration variation; $n_{\mathrm{CH}_{4}}$ and $n_{\mathrm{AIR}}$ is refractive index of methane and air. In our experimental setup, we chose the chamber length of $10 \mathrm{~mm}$, so that the measurement range was $0 \%$ to $10 \%$.

In order to eliminate the influence of dust, water vapor, carbon dioxide, sulfur dioxide, and other gases, the inlet of the sample chamber was resigned as shown in Figure 10(a). 


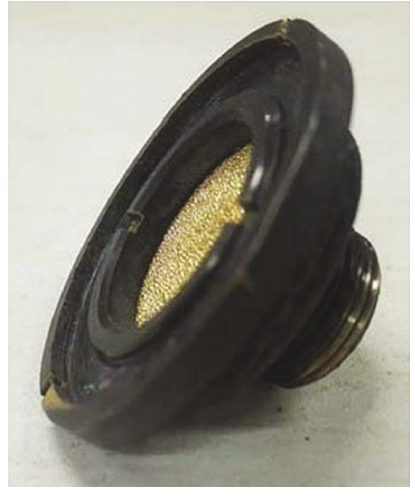

(a)

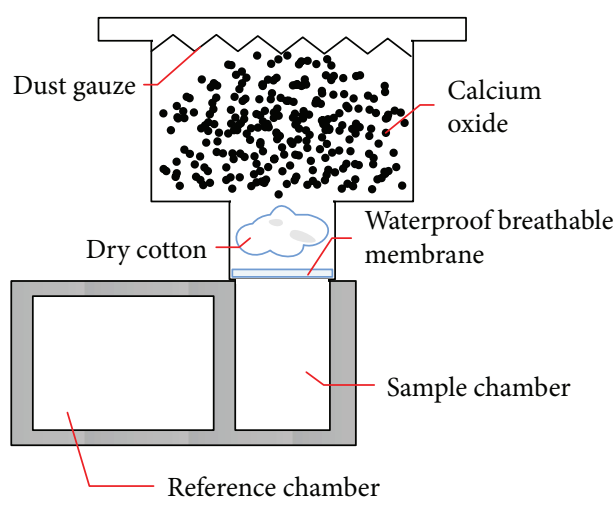

(b)

Figure 10: (a) The inlet of the sample chamber and (b) the inlet section of the sample chamber.

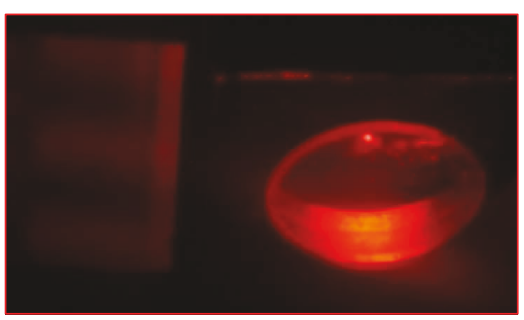

(a)

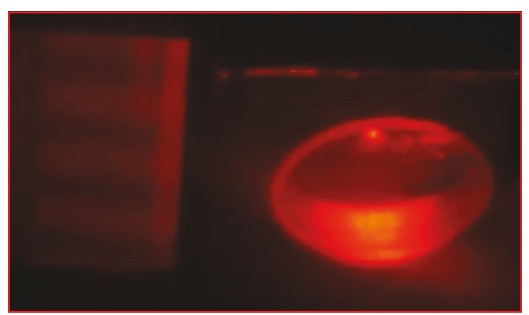

(b)

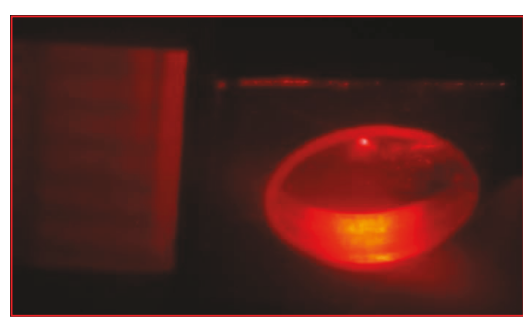

(c)

FIGURE 11: Variation in interference fringe spacing: (a) wide spacing, (b) medium spacing, and (c) narrow spacing.

In the inlet of the sample chamber, waterproof and breathable membrane and chemical reagents such as calcium oxide were used to isolate the external environment. The inlet section of the sample chamber is shown in Figure 10(b).

A temperature control box is used to change ambient temperature, and a vacuum pump is used to generate pressure difference between the reference chamber and the sample chamber to simulate the change of methane concentration. The experimental system is shown in Figure 9(b).

\section{Experimental Result}

Experiments were performed to test the following aspects. First, the included angle $\gamma$ of the compensating prism was changed and the interference fringe spacing was observed. Second, the validity of the temperature drift compensation method was verified.

6.1. Interference Fringe Spacing. The light interference methane sensor was placed in the darkroom, and a white paper was placed in front of the photoelectric detector. The interference fringes fell on the paper because of which the fringe changes could be observed conveniently.

According to the analysis in Section 3.3, the interference fringe spacing can be adjusted by changing the included angle $\gamma$ of the compensating prism. As shown in Figure 11, the variation of the interference fringe spacing is observed under different included angles of the compensating prism. Therefore, the adjustment method proposed in Section 3.3 is feasible and effective.
TABLE 1: Mapping table of methane concentration and output voltage of the photoelectric detector.

\begin{tabular}{lcc}
\hline $\begin{array}{l}\text { Output voltage } \\
(\mathrm{V})\end{array}$ & $\begin{array}{c}\text { Simulated concentration } \\
\text { value }(\%)\end{array}$ & $\begin{array}{c}\text { Pressure difference } \\
(\mathrm{kPa})\end{array}$ \\
\hline 1.650 & -10.0 & 5.18 \\
1.713 & -8.0 & 4.144 \\
1.796 & -6.0 & 3.108 \\
1.895 & -4.0 & 2.072 \\
2.007 & -2.0 & 1.036 \\
2.131 & 0.0 & 0.0 \\
2.263 & 2.0 & -1.036 \\
2.397 & 4.0 & -2.072 \\
2.533 & 6.0 & -3.108 \\
2.667 & 8.0 & -4.144 \\
2.793 & 10.0 & -5.18 \\
2.910 & 12.0 & -6.216 \\
3.015 & 14.0 & -7.252 \\
3.103 & 16.0 & -8.288 \\
3.171 & 18.0 & -9.324 \\
3.220 & 20.0 & -10.36 \\
\hline
\end{tabular}

6.2. Temperature Drift Compensation. According to the temperature drift compensation method described in Section 4.2, the calculation results may be negative due to the light interference detection principle. Therefore, the methane concentration is simulated by the pressure method, and the mapping table is presented in Table 1. 


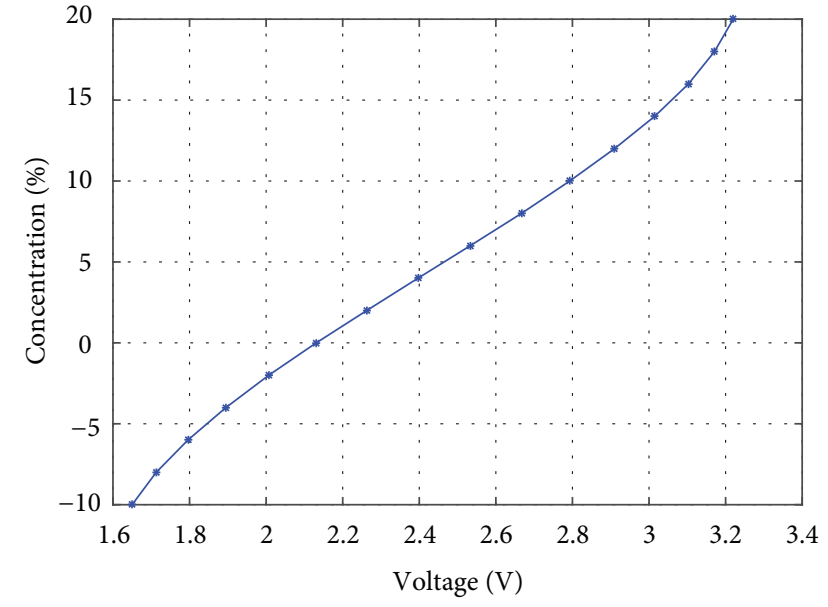

FIGURE 12: Relation between voltage and pressure simulation in mapping table.

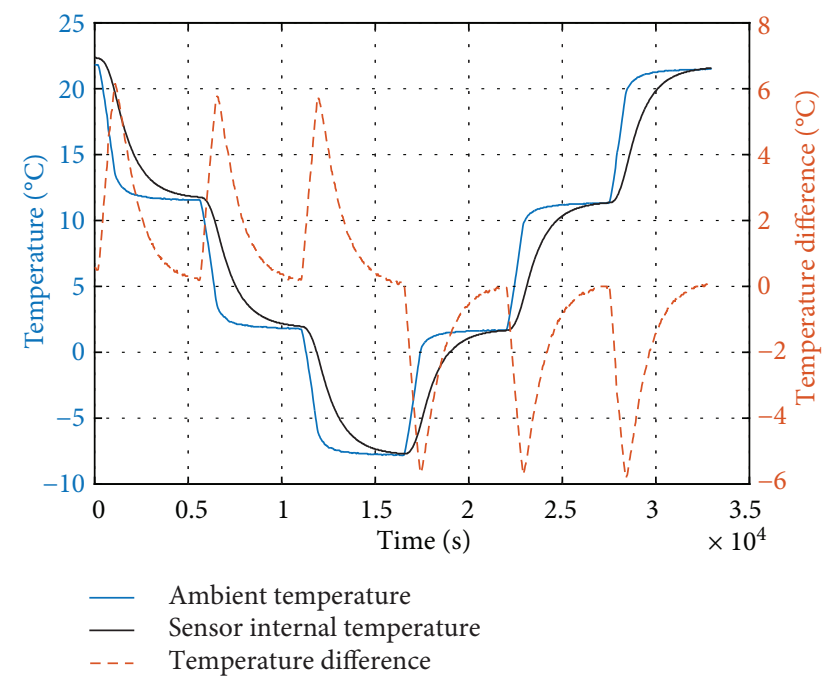

FIGURE 13: Variations in sensor internal temperature, ambient temperature, and temperature difference.

As shown in Figure 12, when the zero drift is greater than $5 \%$, the measuring segment $b c$ will locate the nonlinear segments. In that case, there is a significant error in the segment for high and low methane concentration when the calibration of the mapping table is not used.

The methane concentration is compensated by using the method described in Section 4. As shown in Figure 13, the ambient temperature drops from $20^{\circ} \mathrm{C}$ to $-10^{\circ} \mathrm{C}$ and then rises to $20^{\circ} \mathrm{C}$; therefore, the temperature varies $10^{\circ} \mathrm{C}$ each time in 5000 seconds. The sensor internal temperature is not completely synchronized with the ambient temperature. Therefore, the temperature difference is not constantly zero. As shown in Figure 14, the blue dashed line is a curve of the methane concentration before the compensation. The temperature varies by $10^{\circ} \mathrm{C}$, and the zero drift of the steadystate process is approximately $0.5 \%$. The mapping table and the steady-state compensator are used to compensate for the steady-state process, and the brown dotted line in

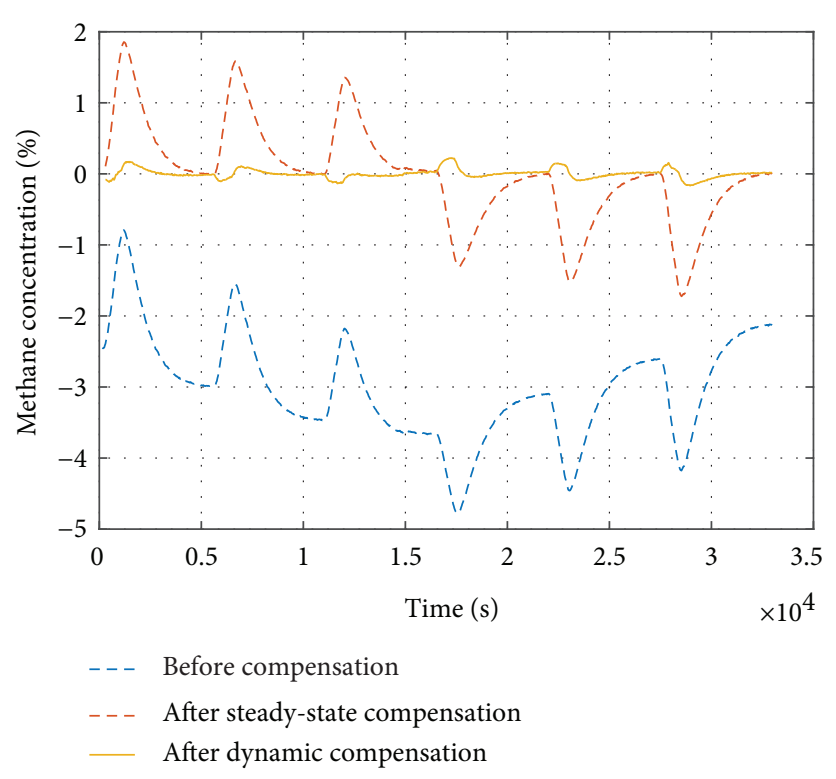

FIGURE 14: Variation in methane concentration with temperature before and after temperature compensation.

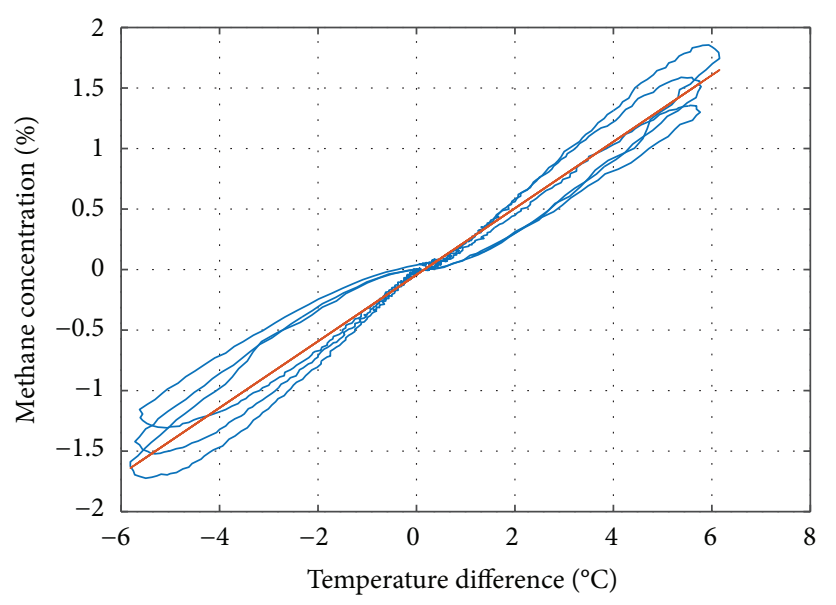

$$
\text { - Actual value }
$$

FIgURE 15: Variation in methane concentration after steady-state compensation with temperature difference.

Figure 14 is obtained. The zero point at the steady-state temperature is almost equal to zero after using the steadystate compensator. However, in the dynamic process of temperature change, the measurement deviation is large, because of which the dynamic compensator should also be used. As shown in Figure 15, the relationship between the temperature difference and the methane concentration after steady-state compensation is approximately linear in this paper, and the primary function is used to fit it. The solid line in Figure 14 is obtained using the fitting function for the compensation of the methane concentration by the steadystate compensator. It meets the precision requirement specified by the standard for $10 \%$ measurement range optical interference methane sensor. 


\section{Conclusion}

A three-dimensional optical path model of the light interference methane sensor based on a point source was proposed in this paper, and the temperature drift was compensated. The main work is summarized as follows:

(1) A three-dimensional optical model of interference methane sensor based on a point source was proposed. This model was closer to actual sensors, and the defects of the planar optical path model are overcome. It could be used to analyze the influencing factors of the interference fringe light intensity distribution and makes the light interference methane sensor more convenient in assembly and adjustment process.

(2) The propagation processes of a single light and multiple lights were analyzed based on the proposed three-dimensional optical path model.

(3) A method for adjusting the interference fringe spacing was proposed. The fringe spacing could be adjusted by changing the included angle of the compensating prism.

(4) A temperature drift compensation method was proposed to improve the measurement accuracy. The method included a mapping table, a steadystate compensator, and a dynamic compensator and it can effectively eliminate the influence of temperature on measurement accuracy.

(5) The experimental setup was designed, and the experimental results could demonstrate the effectiveness of the three-dimensional optical model and the adjustment and compensation methods.

\section{Conflicts of Interest}

The authors claim no conflicts of interest.

\section{Acknowledgments}

This project was supported by the National Natural Science Foundation of China (Grant no. U1713224) and the National Key Research and Development Program of China (Grant no. 2017YFD0701401).

\section{References}

[1] M. van den Bossche, N. T. Rose, and S. F. J. De Wekker, "Potential of a low-cost gas sensor for atmospheric methane monitoring," Sensors and Actuators B: Chemical, vol. 238, pp. 501-509, 2017.

[2] E. E. Karpov, E. F. Karpov, A. Suchkov et al., "Energy efficient planar catalytic sensor for methane measurement," Sensors and Actuators A: Physical, vol. 194, pp. 176-180, 2013.

[3] H. Hadano, A. Miyagi, T. Okuno, Y. Nagawa, and Y. Ishiguro, "Development of a catalytic combustion type gas sensor with low power consumption," ECS Transactions, vol. 75, no. 16, pp. 195-198, 2016.
[4] N. M. Shaalan, M. Rashad, and M. A. Abdel-Rahim, "Repeatability of indium oxide gas sensors for detecting methane at low temperature," Materials Science in Semiconductor Processing, vol. 56, pp. 260-264, 2016.

[5] P. Tardy, J. R. Coulon, C. Lucat, and F. Menil, "Dynamic thermal conductivity sensor for gas detection," Sensors and Actuators B: Chemical, vol. 98, no. 1, pp. 63-68, 2004.

[6] J. Yi, "The research of methane gas sensor based on sno_2," Journal of Transduction Technology, vol. 14, no. 4, pp. 285291, 2001.

[7] P. K. Basu, N. Saha, S. K. Jana, H. Saha, A. L. Spetz, and S. Basu, "Schottky junction methane sensors using electrochemically grown nanocrystalline-nanoporous $\mathrm{ZnO}$ thin films," Journal of Sensors, vol. 2009, Article ID 790476, 9 pages, 2009.

[8] S. Basu and P. K. Basu, "Nanocrystalline metal oxides for methane sensors: role of noble metals," Journal of Sensors, vol. 2009, Article ID 861968, 20 pages, 2009.

[9] L. Liu, B. Xiong, Y. Yan, J. Li, and Z. Du, "Hollow waveguideenhanced mid-infrared sensor for real-time exhaled methane detection," IEEE Photonics Technology Letters, vol. 28, no. 15, pp. 1613-1616, 2016.

[10] S. Zhang, D. Dong, W. Zheng, and J. Wang, "Optical methods for monitoring harmful gas in animal facilities," Optical Engineering, vol. 53, no. 6, article 061602, 2014.

[11] C.-T. Zheng, J.-Q. Huang, W.-L. Ye et al., "Demonstration of a portable near-infrared $\mathrm{CH}_{4}$ detection sensor based on tunable diode laser absorption spectroscopy," Infrared Physics \& Technology, vol. 61, pp. 306-312, 2013.

[12] J. S. Wykes, M. J. Willett, and W. F. Croydon, "Studies of design parameters for interferometric methane sensors," Optical Engineering, vol. 31, no. 8, pp. 1621-1631, 1992.

[13] Y. C. Huang and U. C. Liang, "Interferometric oil-spill detection system," Optical Engineering, vol. 40, no. 5, pp. 740-745, 2001.

[14] S. A. Alexandrov and I. V. Chernyh, "Interference method for determination of the refractive index and thickness," Optical Engineering, vol. 39, no. 9, pp. 2480-2486, 2000.

[15] H. Lin, E. Li, Z. Liang, and B. Zhai, "Configuration improvement and zero-drift error compensation of light interference methane detector," Journal of China Coal Society, vol. 40, no. 1, pp. 218-225, 2015.

[16] H. Lin, Z. Liang, E. Li, M. Yang, and B. Zhai, "Analysis and design of an improved light interference methane sensor," in 11th IEEE International Conference on Control \& Automation (ICCA), pp. 404-409, Taichung, Taiwan, June 2014.

[17] S. Prunet, B. Journet, and G. Fortunato, "Exact calculation of the optical path difference and description of a new birefringent interferometer," Optical Engineering, vol. 38, no. 6, pp. 983-990, 1999. 


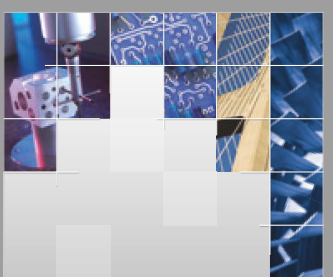

\section{Enfincering}
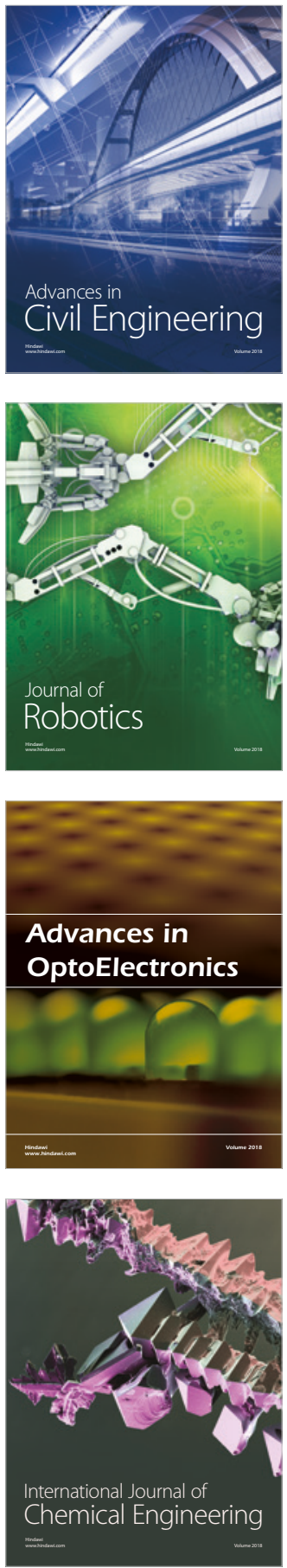

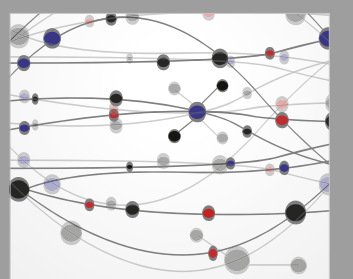

\section{Rotating \\ Machinery}

The Scientific World Journal

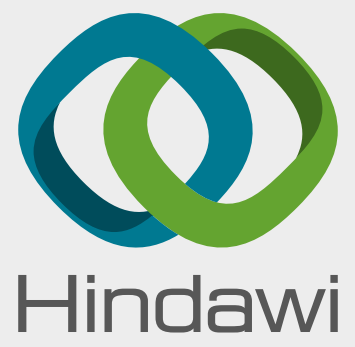

Submit your manuscripts at

www.hindawi.com
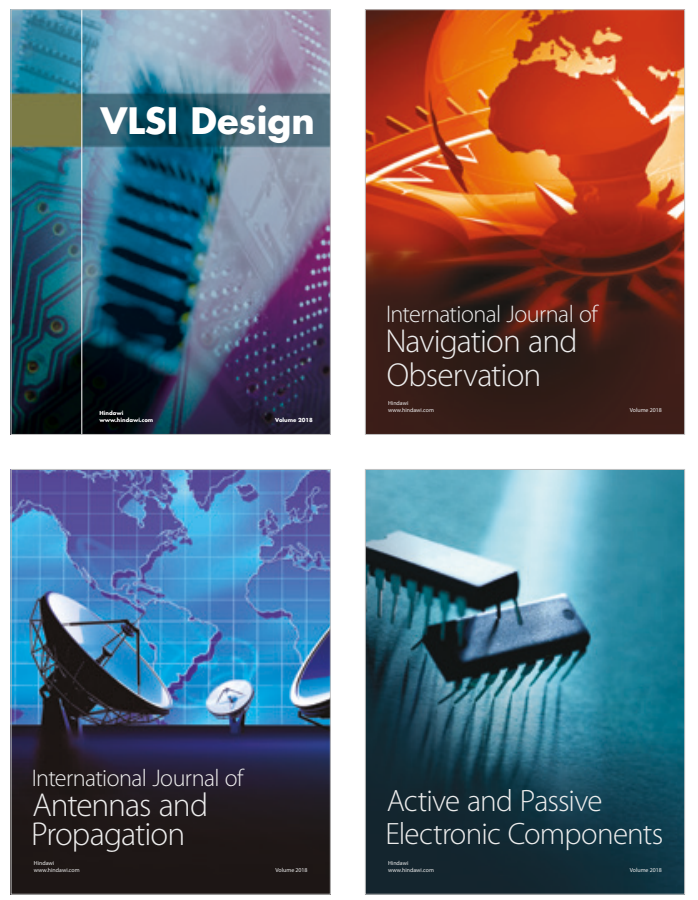
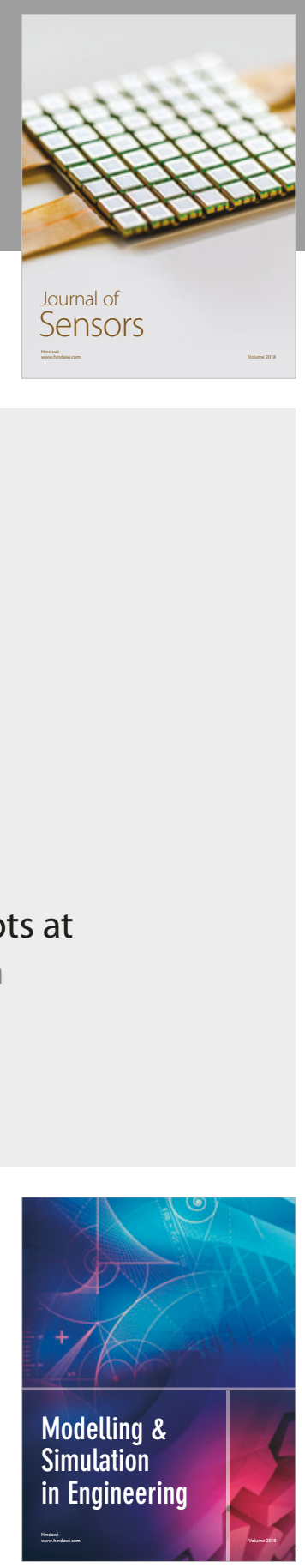

\section{Advances \\ Multimedia}
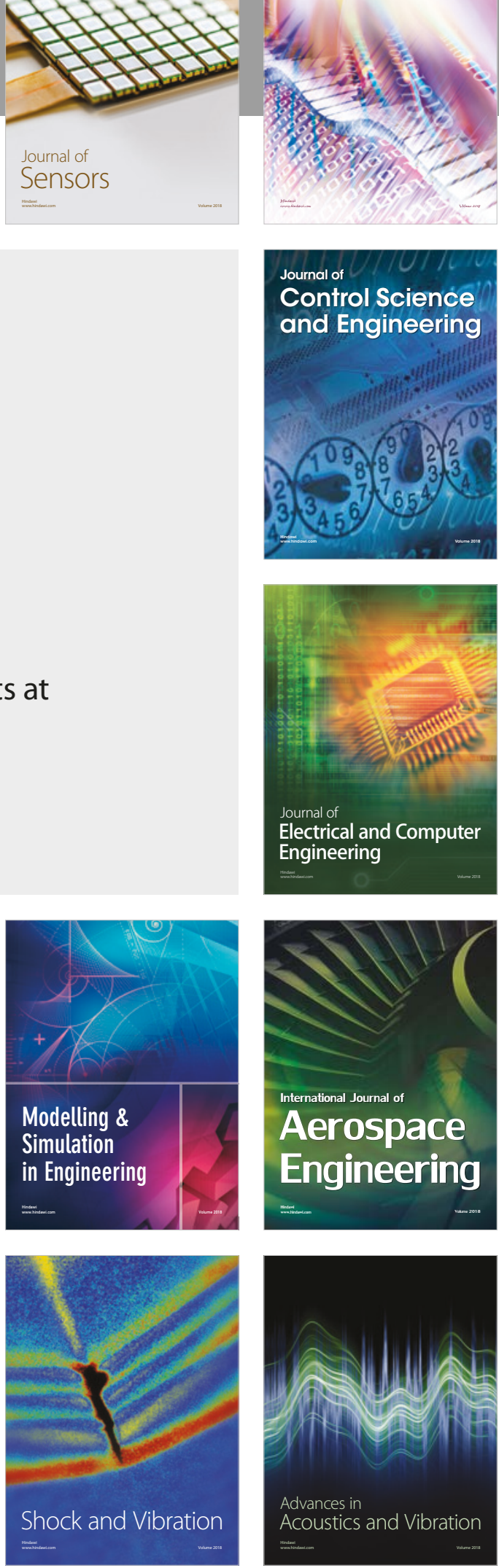